\title{
A protective role for BRCA2 at stalled replication forks
}

\author{
Gurushankar Chandramouly, Nicholas A Willis and Ralph Scully*
}

\begin{abstract}
The hereditary breast and ovarian cancer predisposition genes BRCA1 and BRCA2 account for the lion's share of heritable breast cancer risk in the human population. Loss of function of either gene results in defective homologous recombination (HR) and triggers genomic instability, accelerating breast tumorigenesis. A longstanding hypothesis proposes that BRCA1 and BRCA2 mediate HR following attempted replication across damaged DNA, ensuring error-free processing of the stalled replication fork. A recent paper describes a new replication fork protective function of $B R C A 2$, which appears to collaborate with its HR function to suppress genomic instability.
\end{abstract}

The hereditary breast and ovarian cancer predisposition genes $B R C A 1$ and BRCA2 have crucial roles in the control of double strand break (DSB) repair by homologous recombination (HR). BRCA1 functions early in HR, interacting with nucleases, including CtIP and the Mre11/Rad50/NBS1 (MRN) complex to coordinate DNA end resection to form single-stranded DNA [1]. BRCA1 also recruits BRCA2 to the break site. BRCA2 is a key repair component that loads the Rad51 recombinase onto single-stranded DNA of the processed DSB [2-4]. DSBs in cycling somatic cells are thought to arise predominantly during the DNA synthesis phase of the cell cycle, when a replicative DNA polymerase stalls on abnormal DNA structure. Some models invoke the use of HR to repair the broken fork using the undamaged neighboring sister chromatid as a template [5-7]. However, not all HR requires a DSB intermediate; a fork-stalling DNA adduct may be bypassed without complete breakage ('collapse') of the fork. Because the stalled fork is structurally different from an isolated DSB, the mechanisms governing HR

*Correspondence: rscully@bidmc.harvard.edu

Department of Medicine, Harvard Medical School and Beth Israel Deaconess Medical Center, 330 Brookline Avenue, Boston, MA 02215, USA in these two contexts may differ. Circumstantial evidence suggests that critical events underlying breast cancer risk, as well as the therapeutic action of poly(ADP) ribose polymerase inhibitors on $B R C A$-linked cancers, are played out at sites of DNA polymerase stalling [8-10]. Paradoxically, however, the experimental tools available for studying HR at sites of mammalian DNA polymerase stalling are quite limited.

In a paper published recently in Cell, Schlacher and colleagues [11] have taken an interesting new approach to studying how BRCA2 affects stalled fork metabolism. The authors used single-molecule DNA fiber analysis to determine the fate of newly synthesized ('nascent') DNA strands just proximal to replication forks in cells treated with hydroxyurea $(\mathrm{HU})$, an agent that depletes the nucleotide pool, causing genome-wide DNA polymerase stalling. In wild-type cells, the nascent strands at $\mathrm{HU}$ arrested forks were protected from degradation. In contrast, cells lacking wild-type $B R C A 2$ revealed progressive erosion of nascent DNA strands. The authors identified the DNA end resection complex MRN as a key mediator of this process. Precisely what DNA structures MRN acts on to erode the nascent strands at HU-arrested forks in BRCA2 mutants is not yet clear, although it likely involves MRN-mediated resection of a DNA end. In wild-type cells, the short period of exposure to $\mathrm{HU}$ described by Schlacher and colleagues should produce few if any DSBs as direct products of fork collapse [12]. In this regard, one previous report suggested that $B R C A 2$ mutant cells are particularly susceptible to replication fork collapse following prolonged HU treatment [13]. This raises the possibility that MRN acts on DNA ends produced by fork collapse in $B R C A 2$ mutants. If this were the case, however, only a fraction of all nascent strands should show the observed resection. The fact that Schlacher and colleagues observed coordinated resection of all nascent strands, a process that had evidently begun within minutes of HU exposure, suggests that a mechanism other than fork collapse generated the DNA ends that MRN attacks. Experiments in yeast and bacteria have revealed that stalled forks can undergo 'fork reversal' - a process whereby the fork backtracks on its path, generating a cruciform DNA structure called a 
'chicken foot', with a single exposed DNA end composed of paired nascent strands [14,15]. Schlacher and colleagues speculate that an equivalent structure can form in mammalian cells and that BRCA2 either prevents its formation or helps to protect the free DNA end of this cruciform structure from attack by MRN.

BRCA2 protection of the HU-stalled fork from MRNmediated resection could reflect a non-canonical role for BRCA2/Rad51 at stalled forks. Indeed, Schlacher and colleagues found that a BRCA2 mutant lacking the carboxy-terminal Rad51 binding domain is defective for protection against HU-triggered nascent strand resection, but retains intact HR repair of a chromosomal DSB. These observations, together with other experiments reported in the paper, suggest that BRCA2 has a 'stalled fork-protective' function mediated by its stabilizing effect on the Rad51 nucleoprotein filament but distinct from its role in traditional DSB repair by HR [11]. A caveat of this interpretation is the likelihood that $\mathrm{HR}$ at stalled forks involves mechanisms additional to those required for HR at an isolated DSB. Perhaps the 'forkprotective' function of BRCA2 is indeed required for HR at stalled forks, but not for HR at isolated DSBs. Data obtained with use of $\mathrm{HU}$ also pose some problems of interpretation, since $\mathrm{HU}$ produces genome-wide arrest of otherwise structurally normal replication forks. It is not clear to what extent this static form of 'replication stress' reflects the dynamic interactions that occur between the replication fork and a DNA polymerase-stalling carcinogenic DNA adduct.

Our current view of BRCA1 and BRCA2 suggests that regulation of $\mathrm{HR}$ at stalled replication forks influences the probability of a woman developing breast cancer as well as her response to therapy. The work by Schlacher and colleagues reveals new mechanisms underlying this relationship. The rewards of continuing to dig deeper into basic mechanisms of action of the $B R C A$ genes will be a better understanding of breast cancer risk and breast cancer therapy. Perhaps this will include the discovery of new therapies that take advantage of the replicationrecombination 'Achilles heel' of $B R C A$ mutant breast cancers and of other cancers in which HR is defective.

\section{Abbreviations}

DSB, double strand break; $H R$, homologous recombination; $H U$, hydroxyurea; MRN, Mre11/Rad50/NBS1.
Competing interests

The authors declare that they have no competing interests.

Published: 7 September 2011

\section{References}

1. Ciccia A, Elledge SJ: The DNA damage response: making it safe to play with knives. Mol Cell 2010, 40:179-204.

2. Yang H, Li Q, Fan J, Holloman WK, Pavletich NP: The BRCA2 homologue Brh2 nucleates RAD51 filament formation at a dsDNA-ssDNA junction. Nature 2005, 433:653-657.

3. Jensen RB, Carreira A, Kowalczykowski SC: Purified human BRCA2 stimulates RAD51-mediated recombination. Nature 2010, 467:678-683.

4. Thorslund T, Mcllwraith MJ, Compton SA, Lekomtsev S, Petronczki M, Griffith JD, West SC: The breast cancer tumor suppressor BRCA2 promotes the specific targeting of RAD51 to single-stranded DNA. Nat Struct Mol Biol 2010, 17:1263-1265.

5. Michel B, Boubakri H, Baharoglu Z, LeMasson M, Lestini R: Recombination proteins and rescue of arrested replication forks. DNA Repair (Amst) 2007, 6:967-980.

6. Branzei D, Foiani M: Maintaining genome stability at the replication fork. Nat Rev Mol Cell Biol 2010, 11:208-219.

7. Nagaraju G, Scully R: Minding the gap: The underground functions of BRCA1 and BRCA2 at stalled replication forks. DNA Repair (Amst) 2007, 6:1018-1031.

8. Scully R, Livingston DM: In search of the tumour-suppressor functions of BRCA1 and BRCA2. Nature 2000, 408:429-432.

9. Bryant HE, Schultz N, Thomas HD, Parker KM, Flower D, Lopez E, Kyle S, Meuth M, Curtin NJ, Helleday T: Specific killing of BRCA2-deficient tumours with inhibitors of poly(ADP-ribose) polymerase. Nature 2005, 434:913-917.

10. Farmer H, McCabe N, Lord CJ, Tutt AN, Johnson DA, Richardson TB, Santarosa M, Dillon KJ, Hickson I, Knights C, Martin NM, Jackson SP, Smith GC, Ashworth A: Targeting the DNA repair defect in BRCA mutant cells as a therapeutic strategy. Nature 2005, 434:917-921.

11. Schlacher K, Christ N, Siaud N, Egashira A, Wu H, Jasin M: Double-strand break repair-independent role for BRCA2 in blocking stalled replication fork degradation by MRE11. Cell 2011, 145:529-542.

12. Petermann E, Orta ML, Issaeva N, Schultz N, Helleday T: Hydroxyurea-stalled replication forks become progressively inactivated and require two different RAD51-mediated pathways for restart and repair. Mol Cell 2010, 37:492-502.

13. Lomonosov M, Anand S, Sangrithi M, Davies R, Venkitaraman AR: Stabilization of stalled DNA replication forks by the BRCA2 breast cancer susceptibility protein. Genes Dev 2003, 17:3017-3022.

14. Seigneur M, Bidnenko V, Ehrlich SD, Michel B: RuvAB acts at arrested replication forks. Cell 1998, 95:419-430.

15. Sogo JM, Lopes M, Foiani M: Fork reversal and ssDNA accumulation at stalled replication forks owing to checkpoint defects. Science 2002, 297:599-602.

doi:10.1186/bcr2918

Cite this article as: Chandramouly $\mathrm{G}$, et al:: A protective role for BRCA2 at stalled replication forks. Breast Cancer Research 2011, 13:314. 